\title{
The effects of continuous application of sunscreen on photoaged skin in Japanese elderly people - the relationship with the usage
}

This article was published in the following Dove Press journal:

Clinical, Cosmetic and Investigational Dermatology

26 April 2016

Number of times this article has been viewed

\author{
Makoto Mizuno ${ }^{1,2}$ \\ Kayo Kunimoto' \\ Eiji Naru² \\ Koichi Kameyama ${ }^{2}$ \\ Fukumi Furukawa' \\ Yuki Yamamoto ${ }^{1,3}$ \\ 'Department of Dermatology, \\ Wakayama Medical University, \\ Wakayama, Japan; ${ }^{2}$ Fundamental \\ Research Laboratories, KOSÉ \\ Corporation, Tokyo, Japan; \\ ${ }^{3}$ Department of Cosmetic \\ Dermatology and Photomedicine, \\ Wakayama Medical University, \\ Wakayama, Japan
}

\begin{abstract}
Since photoaging of skin is caused by chronic sun exposure, it is well-recognized that regular sunscreen use can help prevent photoaging of skin in fair-skinned people. Therefore, application of sunscreen is recommended for the prevention of photoaging in many countries. However, the relationship between UV exposure and photoaging has rarely been investigated in clinical studies in Japan. In addition, there have been almost no longterm interventional studies in Japanese people. We have previously conducted a study where Japanese actinic keratosis patients were instructed to continuously apply sunscreen. The results indicated that long-term application of sunscreen is effective in suppressing actinic keratosis progression and generation. In the present study, we investigated the effects of sunscreen on photoaged skin in 14 elderly Japanese people. Skin conditions such as water content, transepidermal water loss, the number of spots, wrinkles, and skin color tone uniformity were measured and compared before and after the study. A statistically significant difference was observed only in skin surface hydration. There were large inter-individual differences in amount of sunscreen used throughout the study. The changes in the number of spots and skin color tone uniformity during the 18 months showed good correlation with amount of sunscreen being used. These results suggest an increase in the number of spots and deterioration in skin color tone uniformity in the 18-month non-sunscreen application period, and that such skin conditions improved with increasing use of sunscreen. In this study, we suggested an inhibitory effect on photoaging symptoms such as spots and skin color tone non-uniformity, by application of the appropriate amount of sunscreen over a long period of time in Japanese people, similar to Caucasians.
\end{abstract}

Keywords: UV, prevention, spot, wrinkle, skin color tone, moisturizing

\section{Introduction}

Photoaging is induced by cumulative sun exposure and is frequently seen in elderly people exposed to a large quantity of ultraviolet (UV) rays. It is well-recognized that regular sunscreen use can help prevent photoaging of skin in fair-skinned people. ${ }^{1-3}$ Therefore, application of sunscreen is recommended for the prevention of photoaging as well as squamous cell carcinoma (SCC) or actinic keratosis (AK) in many countries. In the USA, sunscreen is available over-the-counter. For sunscreens with sun protection factor 15 or higher and broad spectrum UV protection, it is allowed to state on the package insert "not only protects against sunburn, but, if used as directed with other sun protection measures, can reduce the risk of skin cancer and early skin aging". ${ }^{4}$ The European Union (EU) recommendation states that "sunscreen products can prevent the damage linked to photoaging". ${ }^{5}$
Correspondence: Makoto Mizuno Fundamental Research Laboratories, KOSÉ Corporation, I-18-4 Azusawa, Itabashi-ku Tokyo I74-005I, Japan

$\mathrm{Tel}+8 \mathrm{I} 33967644$ I

Fax +81339676649

Email m-mizuno@kose.co.jp Clinical, Cosmetic and Investigational Dermatology 2016:9 95-I05
$\begin{aligned} & \text { (c) } 95 \text { (i) } 2016 \text { Mizuno et al. This work is published and licensed by Dove Medical Press Limited. The full terms of this license are available at https://www.dovepress.com/terms. } \\ & \text { php and incorporate the Creative Commons Attribution - Non Commercial (unported, v3.0) License (http://creativecommons.org/licenses/by-nd/3.0/). By accessing the work }\end{aligned}$ you hereby accept the Terms. Non-commercial uses of the work are permitted without any further permission from Dove Medical Press Limited, provided the work is properly attributed. For

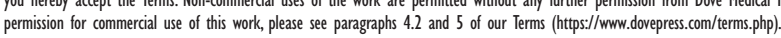


To elucidate the relationship between UV exposure and the development of photoaging in Japanese people, some clinical examinations were conducted. Hillebrand et al performed an epidemiological survey and revealed the relationship between the severity of photoaging skin conditions and latitude of residential area. ${ }^{6}$ Asakura et al evaluated the facial skin aging conditions in 802 Japanese elderly people and identified lifestyle factors associated with skin aging. They concluded that the custom of using sunscreen or cosmetic foundation containing sunscreen was effective in preventing photoaging. ${ }^{?}$ There are also some studies that report the characteristics of photoaging in Japanese people. Comparison of age-related changes of photoaging in Japanese females and Caucasian or other Asian females revealed that Caucasians are more susceptible to wrinkles or skin sagging than Japanese, ${ }^{8,9}$ and there are some differences in characteristics of photoaging even among Asian people. ${ }^{10}$

However, the relationship between UV exposure and photoaging has rarely been demonstrated by interventional studies in Japan. Therefore, it is not approved to advocate the preventive effects of sunscreen cosmetics against photoaging in Japan under the Pharmaceutical Affairs Act, which outlines the use of drugs and cosmetics, and is operated by the Ministry of Health, Labour, and Welfare. In addition, there have also been almost no long-term interventional sunscreen studies which indicate the efficacy of sunscreen in reducing SCC incidence in Japanese. The clinical practice guidelines for malignant skin tumors published by the Japanese Dermatological Association states that the efficacy of UV protection in reducing SCC incidence in most Japanese people remains unclear due to the lack of reliable data implicating UV in SCC development. ${ }^{11}$

Our previous article reported an interventional study where Japanese AK patients were instructed to continuously apply sunscreen for 18 months. No increase in the number of facial AK lesions or worsening of the lesions was observed in the subjects, and dermoscopic observation and immunohistochemical analysis found improvements such as enhanced vascularization. These results indicate that continuous application of sunscreen is effective in suppressing AK progression and generation, and for the prevention of SCC development. ${ }^{12}$

In the present study, we investigated the effects of continuous application of sunscreen for 18 months on photoaging in elderly people.

\section{Methods \\ Study design}

This is a single arm interventional study. It was conducted at the Department of Dermatology, Wakayama Medical University Hospital from November 2010 to November 2013.
This trial was approved by the ethical committee at Wakayama Medical University Hospital and complied with the Declaration of Helsinki guidelines. Written informed consents were obtained from the subjects.

Fourteen elderly Japanese subjects (seven men and seven women; mean age 79.6, range: 62-91 years) were provided with sunscreen products for 18 months. Regarding skin phototype of the subjects, ${ }^{13}$ three subjects were categorized as type II and the other eleven subjects were type III. Study subjects were provided with sunscreen samples during the study period.

\section{Quantity of sunscreen used}

At the beginning of the study, subjects were shown the exact method of application by the attending dermatologist and received an instruction leaflet regarding the appropriate amount $\left(\sim 2 \mathrm{mg} / \mathrm{cm}^{2}\right)$ of application with a sample photograph. The subjects were directed to apply sunscreen on the face and other UV-exposed regions every morning after washing their face.

Six or more $100 \mathrm{~mL}$ bottles of sunscreen were provided free of charge at every 6-month visit for clinical examination. When the residual quantity of the sunscreen became small, additional samples were provided to the subjects. The amount of applied sunscreen was checked and appropriate use was directed at every 6-month visit. The quantity of sunscreen used was estimated by weighing the recovered used sunscreen vessels.

\section{Sunscreen sample}

The sunscreen contains six chemical UV absorbers, 3.95\% (by weight) ethylhexyl methoxycinnamate, 2\% (by weight) bis-benzotriazolyl tetramethylbutylphenol, $1.05 \%$ (by weight) diethylamino hydroxybenzoyl hexyl benzoate, $1 \%$ (by weight) bis-ethylhexyloxyphenol methoxyphenyl triazine, $1 \%$ (by weight) ethylhexyl triazone, and $1 \%$ (by weight) methylene phenylbenzimidazole sulfonic acid. The sunscreen exhibits broad spectrum protection against both UVA and UVB rays. The spectrum of the sunscreen is shown in Figure 1. Sun protection factor of the sunscreen is 30.4. UVA protection factor of the product value, a factor for UVA protection efficacy is 8.0 .

\section{Clinical evaluations}

Clinical evaluations of skin symptoms were performed at 0 , 6,12 , and 18 months by a dermatologist. Regarding skin surface conditions, stratum corneum hydration, transepidermal water loss (TEWL), and skin color tone uniformity were measured non-invasively at 0 and 18 months. Stratum 


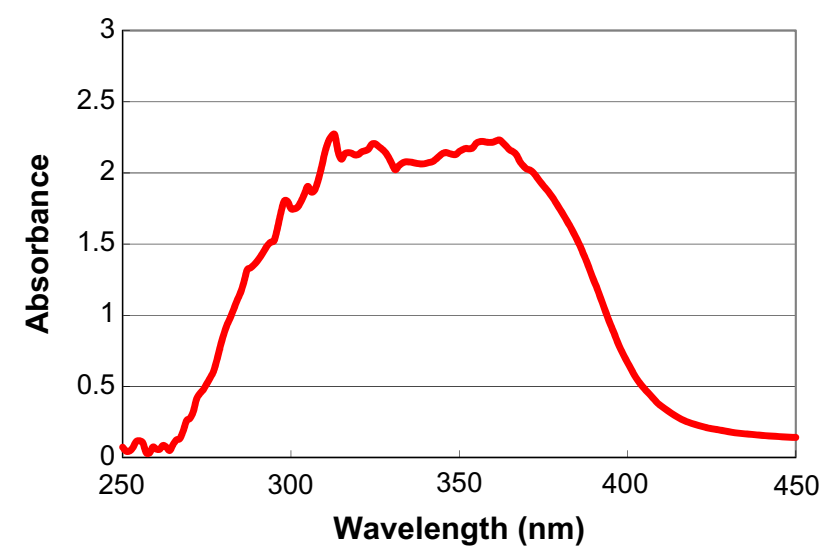

Figure I Absorption spectra of the sunscreen.

Notes: Absorption spectra of the sunscreen was measured using a spectrometer (UV-2000S; Labsphere, Inc., North Sutton, NH, USA). The absorbance was measured in ultraviolet light and a part of visible light wavelength region from 250 to $450 \mathrm{~nm}$. The wavelength increment step was I $\mathrm{nm}$.

corneum hydration and TEWL on the cheek were measured non-invasively (Corneometer ${ }^{\circledR}$ CM825 and Tewameter ${ }^{\circledR}$ TM 300; Courage+Khazaka electronic GmbH, Cologne, Germany). The subjects' faces were photographed using a digital image analysis system (Visia ${ }^{\circledR}$ Complexion Analysis; Canfield Scientific, Inc., Fairfield, NJ, USA). The number of pigmentation spots and wrinkles on the face were counted using the pictures. The skin color tone uniformity was assessed by detecting uneven skin tone area of the face. The uneven skin tone area was defined as significantly different color compared to peripheral skin. The photographs were analyzed in a pixel unit and the pixels with significantly different color compared to peripheral skin were counted as uneven skin tone. The areas of the uneven skin tone were calculated using the image analysis system.

\section{Statistical analysis}

The differences in skin parameters at the beginning and the end of the study were analyzed using a paired Student's

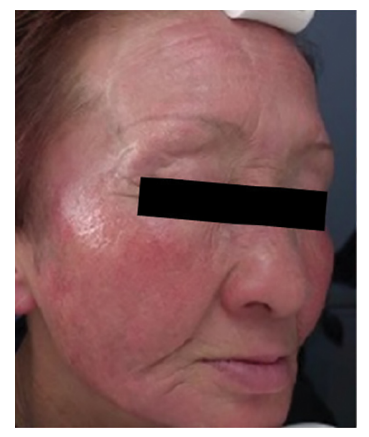

0 months

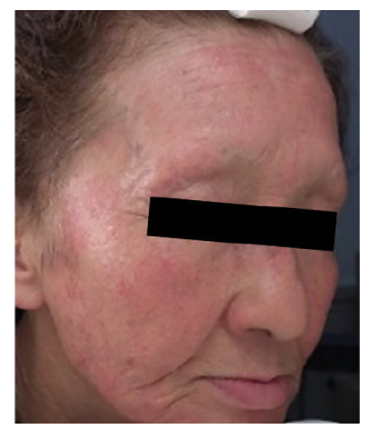

6 months

$t$-test. The strength of the association between the two variables was calculated using the Pearson correlation coefficient. Simple linear regression equations were calculated using the method of least squares. Tests were two-sided and results were considered statistically significant when $P<0.05$. All statistical analyses were conducted using commercial software JMP version 9.0 (SAS Institute Inc., Cary, NC, USA).

\section{Results \\ Clinical appearance}

Severe photoaging symptoms such as spots and wrinkles were observed in the subjects of this study, likely because of advanced age; all the subjects were 62 years or older with average age 79.6 years. Stratum corneum thickness and flaky conditions were observed in many subjects during clinical examination performed at the start of the study. Mild erythema was observed in some subjects, likely due to chronic UV exposure.

The subjects were asked to attend follow-up visits every 6 months for clinical examination, and we found that the skin color gradually became brighter in many subjects. In some subjects, remarkable improvements in stratum corneum thickness and flaky conditions on their whole face were observed.

The medical interviews at the end of the study revealed that many subjects reported improvements in the smoothness of their skin surface.

Clinical photographs of one subject taken every 6 months from the beginning of the study are shown in Figure 2. She had experienced symptoms of erythema on her entire face. These photographs indicate that the erythema conditions on her face were gradually suppressed; the cheek, top of the nose, and forehead, all severely UV-irradiated parts, were remarkably improved.

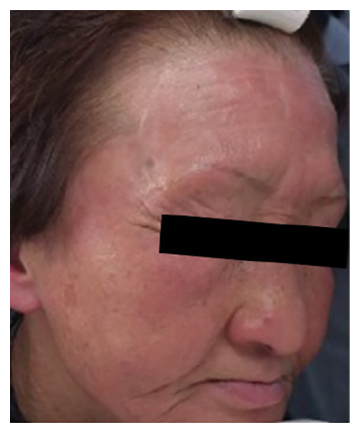

12 months

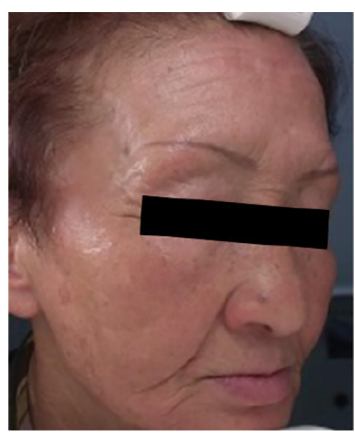

18 months

Figure 2 Clinical appearance of a subject at 6-month intervals.

Notes: Clinical evaluations of skin symptoms were performed at 0, 6, I2, and I8 months. These photographs were taken by a digital image analysis system (Visia ${ }^{\circledR}$ Complexion Analysis; Canfield, NJ, USA) 
No adverse events related to the test material occurred during the study.

\section{The change in skin conditions}

Skin conditions of the subjects were compared before and after the 18-month application of the study material. The changes in skin parameters are shown in Figure 3A-E. A statistically significant difference was observed in skin surface hydration. No statistically significant differences were demonstrated before and after sunscreen application in TEWL, the number of spots, the number of wrinkles, or the uneven skin tone area. These findings suggest that the water content of the stratum corneum increased after the study.
In addition, spots, wrinkles, and skin color tone uniformity did not worsen, but retained their pre-study state.

Regarding the values of these skin parameters or the 18-month change, a statistically significant difference between men and women or the groups by skin phototype was not observed (data not shown).

In this study, symptom progression could not be compared between sunscreen-applied and non-applied (control) groups because a control group could not be established due to ethical reasons. No statistically significant improvements were demonstrated in the sunscreen-applied group in photoaging parameters such as the number of spots and wrinkles and uneven skin tone area. Therefore, while the unchanging
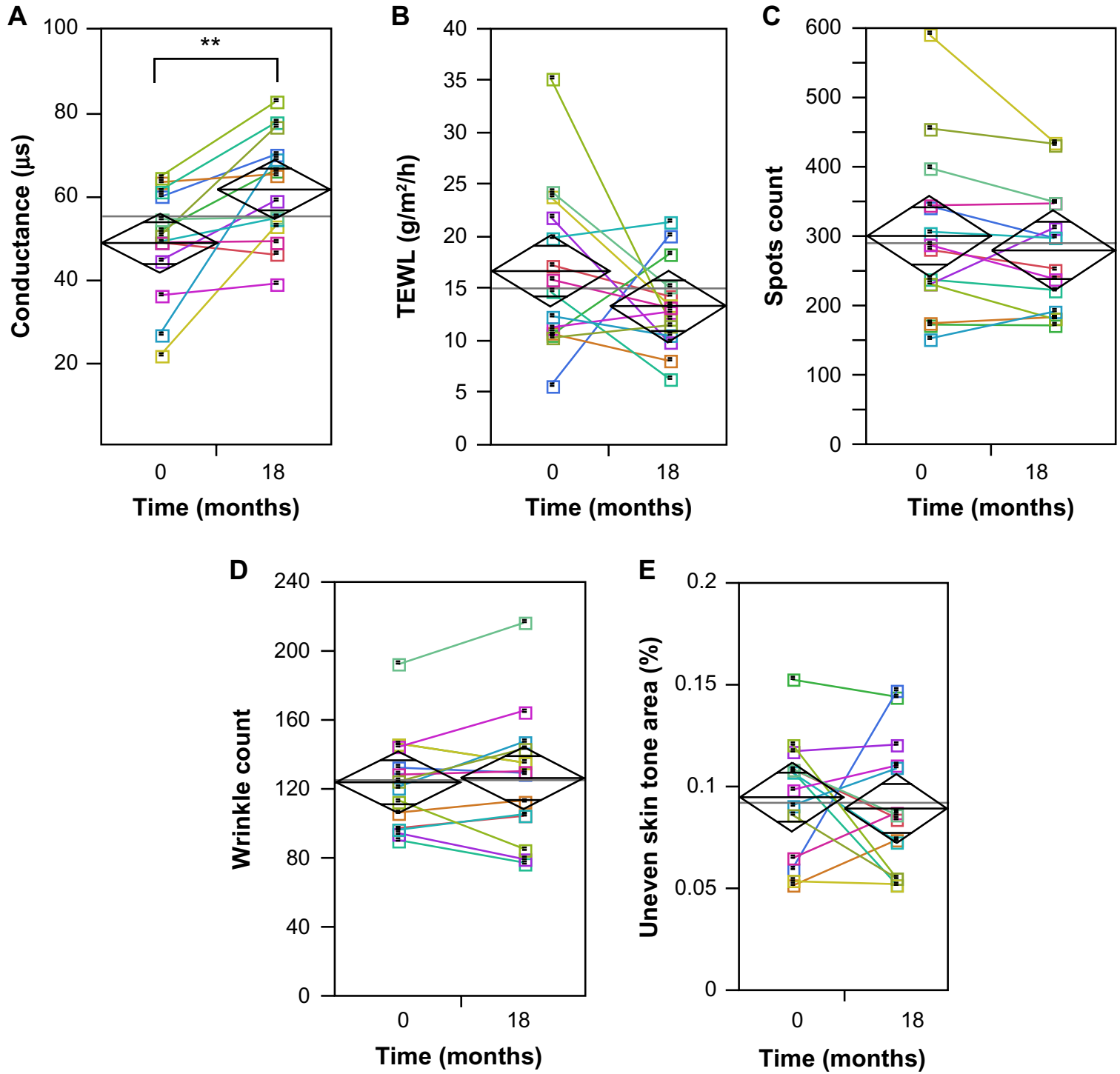

Figure 3 Skin conditions of the subjects at the beginning and the end of the clinical study.

Notes: Conductance $(\mathbf{A})$, TEWL $(\mathbf{B})$, spot count $(\mathbf{C})$, wrinkle count $(\mathbf{D})$, and uneven skin tone area $(\mathbf{E})$ before and after the study. The data at 0 and I 8 months of the same subject were connected with a straight line. Center lines of bold black rhombuses indicate the average, top and bottom of the rhombuses indicate $95 \%$ confidence intervals. The differences in skin parameters between 0 and 18 months of the study were analyzed using a paired Student's $t$-test. A statistically significant difference was observed only in conductance $(* * P<0.01)$. No significant differences were shown in the other parameters. Each colored line represents a different subject.

Abbreviation: TEWL, transepidermal water loss. 
conditions observed over the 18 months did not disprove the effect of treatment in inhibiting photoaging symptom progression, concrete evidence directly indicating that continuous application of sunscreen clearly inhibits periodic progress of photoaging symptoms could not be obtained.

\section{Sunscreen use}

Individual amounts of sunscreen used throughout the study is shown in Figure 4. There seemed to be a large difference in the amount of sunscreen used, and the largest amount (63.7 g/month) applied was approximately 9.8 times more than the smallest amount $(6.47 \mathrm{~g} /$ month $)$. Average amount used was $30.1 \pm 15.0 \mathrm{~g} / \mathrm{month}$ in all the subjects, $31.7 \pm 17.0 \mathrm{~g} / \mathrm{month}$ for men, and $28.6 \pm 12.9 \mathrm{~g} / \mathrm{month}$ for women. Differences between men and women were not seen. Estimated amount used was $35.3 \mathrm{~g} /$ month for men, $34.4 \mathrm{~g} / \mathrm{month}$ for women when applying our recommended amount of $2 \mathrm{mg} / \mathrm{cm}^{2}$ of the sample on the whole face every day. ${ }^{14}$ As the sunscreen was also applied to other parts of the body like the hands, substantial average application amount was thought to be less than our recommended amount.

These results indicate that there were subjects who were almost adequately, and inadequately protected from UV exposure. However, they cannot be compared because total UV exposure time and exposed skin areas varied among the subjects.

\section{Correlations between changes in skin parameters and sunscreen use}

In this study, symptom progression could not be compared between sunscreen-applied and non-applied groups because

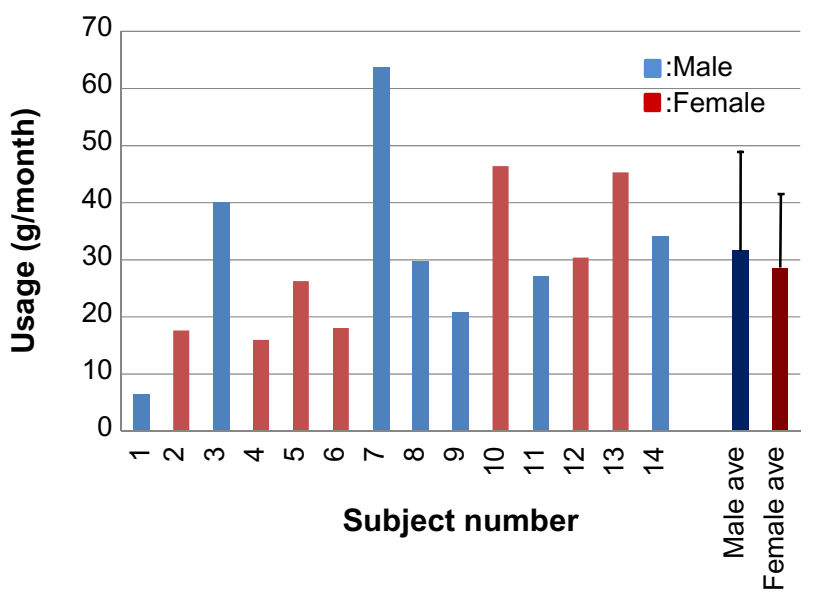

Figure 4 Amount of sunscreen used by each subject.

Notes: Light blue columns and light red columns indicate male and female subjects respectively. Dark blue and dark red columns with error bars indicate the average (ave) amount \pm standard deviation of male and female subjects respectively $(n=7)$. a non-applied control group was not established. However, since the amount of sunscreen used varied widely between individuals, we hypothesized that the effect of long-term application of sunscreen may vary depending on amount being used.

Therefore, the correlation between changes in each skin parameter and sunscreen use during the 18 months was investigated (Figure 5A-E). All approximate expressions for the relationship between sunscreen use and the parameters indicated that skin condition improved as more sunscreen was used. Statistical correlations $(R)$ were 0.51 , $-0.24,-0.68,-0.20$, and -0.80 as shown in Figure $5 \mathrm{~A}-\mathrm{E}$, respectively. Specifically, improvements in uneven skin tone area and spots showed good correlation with amount of sunscreen used.

Y-intercept and X-intercept values were calculated from approximate expressions of uneven skin tone area, number of spots, and water content of the skin. The Y-intercept showed changes in the parameters in the cases where sunscreen was not applied for 18 months, while the X-intercept indicated the theoretically calculated amount of sunscreen required to keep the relevant skin parameters unchanged for 18 months.

Y-intercept and $\mathrm{X}$-intercept values for uneven skin tone area were $0.036 \%$ and $26.7 \mathrm{~g} / \mathrm{month}$, respectively. This indicates that no sunscreen application for 18 months would likely result in the increasing of uneven skin tone area, and that the degree of worsening would be detectable by the measurement method used in this study. Condition of skin color tone uniformity could theoretically be maintained by application of $26.7 \mathrm{~g} / \mathrm{month}$ of sunscreen.

Y-intercept and $\mathrm{X}$-intercept values for the number of spots were 52.4 and $21.6 \mathrm{~g} /$ month, respectively. This means that the number of spots would increase by 52.4 during 18 months without sunscreen and that $21.6 \mathrm{~g} /$ month of sunscreen would be needed to maintain the baseline number of spots.

Water content of the skin, which showed a statistically significant increase, also correlated with the amount of sunscreen applied, although the correlation was not statistically significant $(P=0.06)$. Y-intercept and $\mathrm{X}$-intercept values for water content were $-0.51 \mu \mathrm{S}$ and $1.14 \mathrm{~g} /$ month, respectively. The Y-intercept value of $-0.51 \mu \mathrm{S}$ implies that the degree of decrease in water content, even after 18 months of no sunscreen application, would be limited to within the margin of error. Also, this result indicated that water content could be maintained by application of very small amounts (1.14 $\mathrm{g} /$ month) of sunscreen. Essentially, it means that the water content would increase even if the amount of sunscreen applied is small, and that the more sunscreen is applied, the more the water content would increase. 

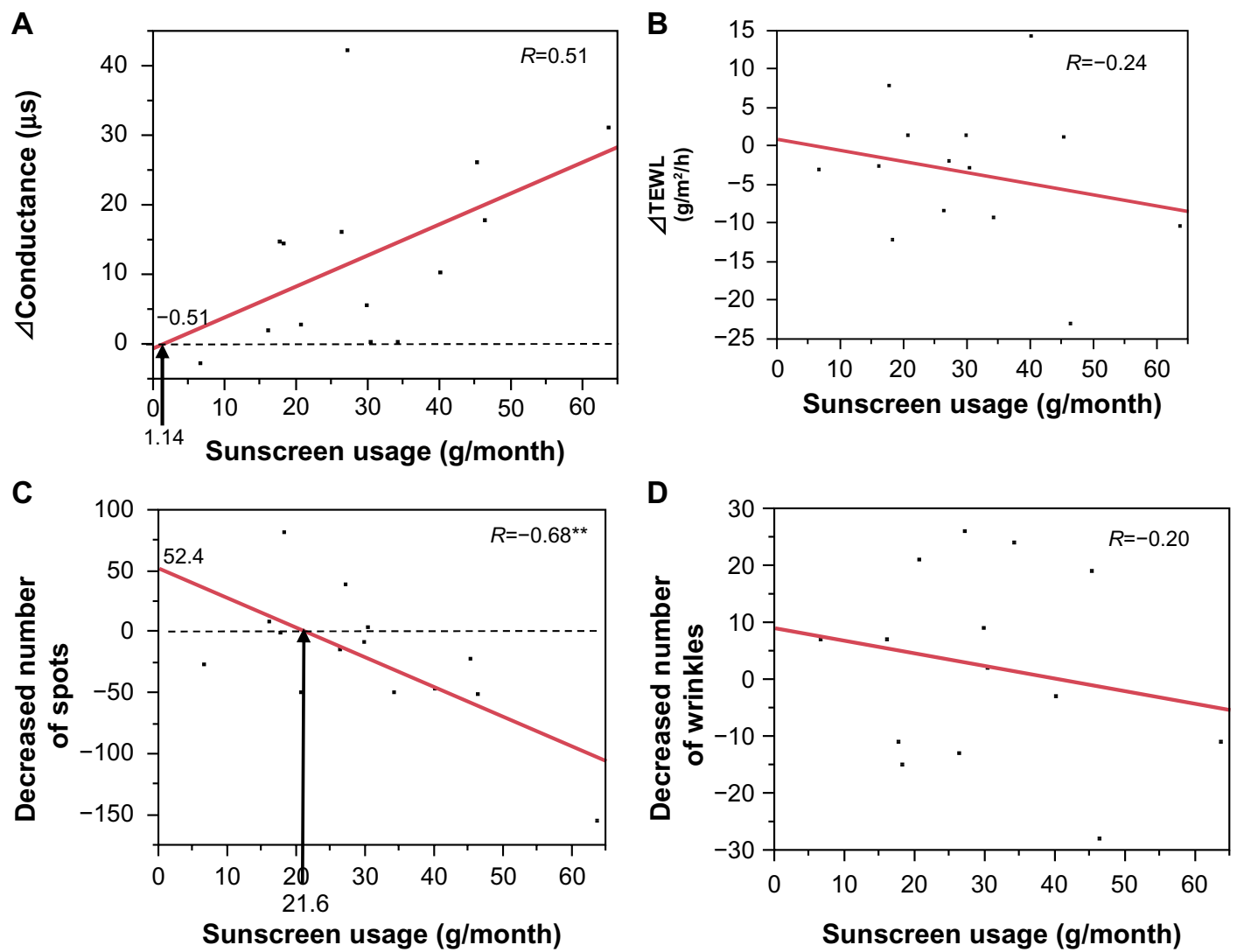

Sunscreen usage (g/month)

Sunscreen usage (g/month)

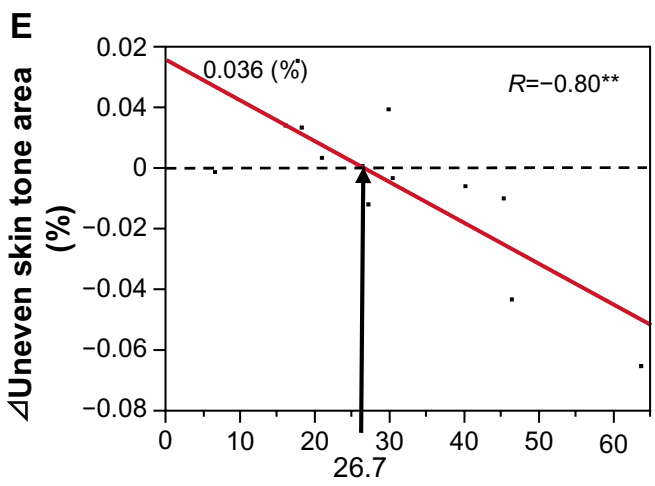

Sunscreen usage (g/month)

Figure 5 Correlation between sunscreen use and changes in skin parameters during 18 months.

Notes: Relation between amount of sunscreen used during 18 months with conductance (A), TEWL (B), number of spots (C), number of wrinkles, (D) and uneven skin color tone area $(\mathbf{E})$. Red lines indicate simple linear regression equations calculated by the method of least squares. Dotted lines indicate $Y=0$. The Pearson correlation coefficient $(R)$ was $0.5 \mathrm{I}(\mathbf{A}),-0.24(\mathbf{B}),-0.68(P<0.0 \mathrm{I})(\mathbf{C}),-0.20(\mathbf{D})$, and $-0.80(P<0.0 \mathrm{I})(\mathbf{E})$ respectively. $Y$-intercept and $\mathrm{X}$-intercept values of approximate expressions are $-0.5 \mathrm{I} \mu \mathrm{S}$ and $1.14 \mathrm{~g} /$ month for conductance (A), 52.4 and $21.6 \mathrm{~g} / \mathrm{month}$ for decreased number of spots (C), $0.036 \%$ and $26.7 \mathrm{~g} / \mathrm{month}$ for uneven skin tone area (E) respectively.

Abbreviation: TEWL, transepidermal water loss.

\section{Discussion}

\section{Symptomatic improvements in clinical appearance}

Clinical examination at the end of the study revealed brightening in skin color as a result of decreased melanin production in the epidermis, suggesting that UV exposure had been reduced during the study period.
Stratum corneum thickness and flaky skin conditions were found in some subjects at the start of the study. These are symptoms frequently seen in dry skin conditions. ${ }^{15-17}$ Elderly people often present with dry skin conditions such as senile xerosis. A decrease in the water content of the stratum corneum is a skin characteristic of elderly people and is one of the features of aging. ${ }^{18-21}$ Because of this, it is considered that many of the subjects had a dry skin condition at the start 
of the study. Their dry skin conditions were alleviated as the water content of the stratum corneum increased after application of sunscreen, which resulted in improvement in stratum corneum thickness and flaky skin conditions by the end of the study. The improvement in skin texture experienced by many subjects after the study likely resulted from these changes.

The subject shown in Figure 2 exhibited severe inflammatory conditions especially in the UV-exposed areas at the start of the study, but this improved during the study period. Therefore, it was considered that the UV-induced chronic inflammatory conditions found in the subject at the start of the study were mitigated by UV protection. Chronic inflammatory conditions are indicated to accelerate skin aging and increase risk of cancer development. ${ }^{22-24}$ Thus, application of sunscreen may result in inhibition of photoaging or photocarcinogenesis in subjects who show mitigation of inflammatory conditions. In fact, after 18 months of sunscreen application, the subject clearly showed improvements in her symptoms: an increase of $83.1 \mu \mathrm{S}$ in the water content, a $23.1 \mathrm{~g} / \mathrm{m}^{2} / \mathrm{h}$ decrease in TEWL, a decrease of 51 in the number of spots, a decrease of 28 in the number of wrinkles, and a $0.043 \%$ decrease in uneven skin tone area.

\section{The effect of skin surface hydration on photoaging}

Several skin parameters were measured before and after the study; only the water content in the stratum corneum showed a statistically significant increase.

The sunscreen used in this study contained approximately $10 \%$ polyalcohol, such as glycerin and butylene glycol, and water-retentive water-soluble polymers. Cosmetics containing polyalcohol such as glycerin are known to have a restorative effect on water content in the stratum corneum. ${ }^{15,16,25,26}$ Many of the commercially available sunscreens, like the sunscreen preparation used in this study, contain ingredients for moisturization in their formulations, which are expected to exert a moisturizing effect. Because the sunscreen used in this study has moisturizing activity as well as UV protection, the study subjects were considered both moisturized and UV-protected during the study period. Some studies and the UK Guidelines for AK report the effectiveness of moisturization for prevention of AK. ${ }^{27-29}$ It is highly likely that an increase in the water content of the stratum corneum due to application of sunscreen is a contributing factor in inhibiting photoaging as well as skin cancer.

\section{Change in skin barrier function}

It is reported that TEWL is increased by one minimal erythema dose of UV irradiation. ${ }^{30}$ We supposed that increased
TEWL by UV exposure would be lower after UV protection using sunscreen in the study. However, TEWL values of the subjects were constant throughout the study period.

Initial TEWL values of the subjects were not as high as expected. Many subjects had normal TEWL values between 10 to $20 \mathrm{~g} / \mathrm{m}^{2} / \mathrm{h}^{31}$ and a few subjects had lower or higher TEWL values than at the beginning. The subjects with higher TEWL values had decreased barrier function caused by dry or inflammatory conditions. On the other hand, it is considered that the transpiration of the skin water content was hampered by stratum corneum thickness caused by chronic UV exposure in the subjects with lower TEWL values.

Correlation between initial TEWL values and changes in TEWL values during the 18 months is shown in Figure 6. It indicates the tendency that subjects with greater TEWL values and low skin barrier function at the start of the study showed decreased TEWL, and conversely, subjects with low values at the start showed increased TEWL values. These results suggest that long-term application of sunscreen restores TEWL values outside the normal range back to the normal range. Therefore, some abnormal degrees of skin barrier function were improved to more normal conditions by long-term application of sunscreen.

\section{Maintenance or improvement in skin color tone uniformity}

A statistically significant correlation was demonstrated between amount of sunscreen used and improvement in uneven skin color tone area. Uneven skin color tone area corresponds to the region where melanin is disproportionately

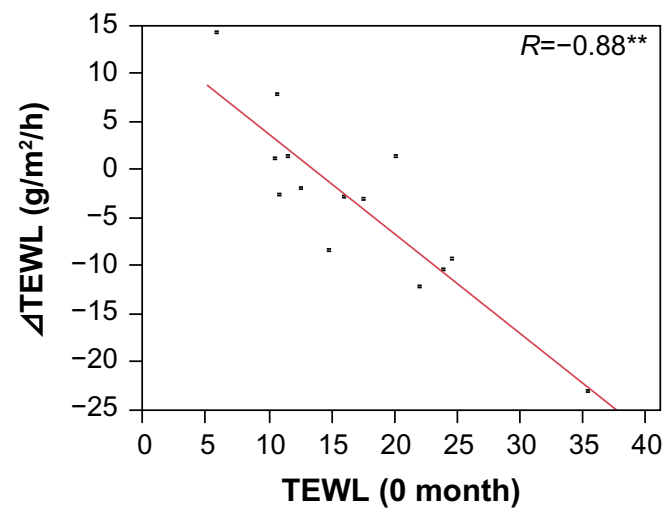

Figure 6 The correlation between initial TEWL values and the variation of TEWL values.

Notes: Relation between TEWL values at 0 month (start of the study) and the change of TEWL values during the 18-month study period is shown. Red lines indicate simple linear regression equations calculated by the method of least squares. The Pearson correlation coefficient $(R)$ was $-0.88(* * P<0.0 \mathrm{I})$.

Abbreviation: TEWL, transepidermal water loss. 
produced and distributed, and also the deteriorated skin texture region where stratum corneum thickness or flaky skin conditions occur. Since skin color non-uniformity or deterioration of skin texture is one of the characteristics of photoaging, ${ }^{32,33}$ the result of maintaining or decreasing uneven skin tone area means suppression or improvement of photoaging. Decreased water content in the stratum corneum causes skin thickness and flaky conditions. ${ }^{15-17}$ UV exposure causes not only uneven melanin distribution, but also increased thickness of the stratum corneum. ${ }^{34}$ Therefore, UV protection and skin moisturization suppress dryness and are able to maintain or improve skin color tone uniformity. Sunscreens with UV protection and moisturizing activity are quite effective in maintaining skin color tone uniformity.

\section{Unchanging number of wrinkles}

Fine wrinkles usually develop after 20 or 30 years because of the deepening of the sulci cutis in dry skin, but they may disappear in the early stage of development by recovering the water content of the stratum corneum. ${ }^{35-37}$ Since a statistically significant increase in the water content in the stratum corneum was demonstrated in the study, we expected the disappearance of fine wrinkles and a decrease in the total number of wrinkles. However, the total number of wrinkles did not statistically significantly decrease or increase 18 months after application, and no association was demonstrated between individual changes in the number of wrinkles and any other factors, including the amount of sunscreen used. This may be explained by the fact that most of the subjects involved in the present study were elderly and showed severe symptom progression in terms of photoaging with deep and large wrinkles. It is difficult to clear up these wrinkles by improving the water content of the stratum corneum, because such wrinkles affect the structure of collagen and elastin in the dermal layer. ${ }^{32}$ Effectiveness of sunscreen on wrinkles of elderly people may be assessed by qualitative characteristics such as depth or length, but not number of wrinkles.

Only younger participants under 55 years old were used for photoaging analysis in a huge clinical trial in Nambour. ${ }^{2,3}$ The authors explained that the reason for this restriction is because skin aging under the age of 55 years is caused predominantly by photoaging, rather than by photoaging and aging. All the subjects in the study were over 60 years. It is thought that a long-term clinical study in approximately 40- to 50-year-old Japanese subjects would be able to prove the effects of sunscreens on wrinkles in the Japanese.

\section{Amount of sunscreen used for prevention of photoaging}

Amount of sunscreen used showed a statistically significant correlation with changes in the number of spots and uneven skin color tone area during the 18 months. These results suggest that sunscreen is effective in a dose-dependent manner, in prevention of photoaging in terms of spots and skin color tone non-uniformity. Approximate expressions show that sunscreen application of 21.6 or $26.7 \mathrm{~g} /$ month is theoretically needed to maintain the number of spots or skin color tone uniformity. The average amount used in this study was more than these values. It indicates that continuous application of average amounts of this sunscreen is adequate to maintain the number of spots and skin color tone uniformity. And it means that the disappearance of spots and improvement in skin color tone uniformity can be expected with increased amounts of sunscreen application.

\section{Adherence to the amount of sunscreen applied}

There appeared to be a big difference in the amount of sunscreen applied among the subjects. There appeared to be a bigger difference in the amounts of sunscreen used by men than women. However, the amount being used was almost the same between them. In Japan, sunscreen is considered a cosmetic product and not an over-the-counter product, and many people, especially aged individuals, regard sunscreen application as applying makeup. Many Japanese women, when compared to men, prefer fair skin and use more sunscreen on a routine basis. Since many aged individuals were involved in this study, it was predicted before the study that less sunscreen would be applied in men compared to women. Our recommended amount of sunscreen in this study was $2 \mathrm{mg} / \mathrm{cm}^{2}$, which is much more than the amount of commonly used cosmetic cream or milk emulsion. It is thought that many female subjects applied the study material according to their personal experiences with using cosmetics. In male subjects, on the other hand, it was thought that some subjects regarded the sunscreen as a cosmetic product and were therefore reluctant to use it, but others who did not regard the sunscreen as a cosmetic product complied with our instructions about the amount used.

The amount of sunscreen commonly used is reported to be 0.3 to $1.3 \mathrm{mg} / \mathrm{cm}^{2} .{ }^{38-42}$ The amount used would be approximately 5.6 to $24.5 \mathrm{~g} /$ month when the subjects used the sunscreen on the entire face every day at this density. ${ }^{14}$ All of these reports conclude that decreased use is directly linked to a reduction in UV protection efficacy on one level 
or another. Poor adherence to treatment frequently results in deterioration in many diseases. ${ }^{43-46}$

There are many sunscreen products in the market, some with better UV protection efficacy than the sunscreen we used in the study. An insufficient application amount prevents the product from demonstrating its full UV protection and photoaging prevention capabilities. To achieve the maximum effect of the sunscreens, guidance on sunscreen use regarding the appropriate amount, application area, and methods is necessary. In addition to these instructions, it is necessary for a dermatologist or other specialist to educate ordinarily healthy people about the increased risk of photoaging and skin cancer due to UV exposure, and that application of sunscreen with the appropriate method and time course may decrease UV exposure and be effective in prevention or improvement of such conditions.

\section{Susceptibility to photoaging in Japanese population}

In our previous study, AK was analyzed and statistically significant improvements were demonstrated in dermoscopy analysis and the expression level of CD31, and slight improvements were revealed in the AK grade in some individuals. These results indicated the preventive effect of sunscreen against AK and SCC in Japanese people. ${ }^{12}$ In Caucasians, many reports demonstrate the disappearance of AK after long-term application of sunscreen. ${ }^{47,48}$ However, in our previous study, no statistically significant difference was observed in the number of AK lesions before and after application of sunscreen, and the number of AK lesions decreased in only a small proportion of the subjects. ${ }^{12}$ It is reported that more marked wrinkle formation and skin sagging are observed in Caucasian females compared to similar aged Japanese females. ${ }^{8}$ When compared to Caucasians, Japanese are thought to have a lower sensitivity to UV and less susceptibility to photocarcinogenesis and photoaging because they have a lower incidence of AK and SCC, and characteristics of lower risk factors for AK such as lower skin phototypes or darker eye colors. ${ }^{49}$ It means that the effect of sunscreens on prevention of photoaging in Japanese might be smaller than that in Caucasians.

However, some reports indicated that pigmentation spots are observed much more frequently and earlier in Japanese or Asians than in Caucasians. ${ }^{9}$ All photoaging symptoms are not necessarily more severe in Caucasians than in Japanese. There was a dose-dependent decrease in the number of spots although no changes in wrinkles were seen during the 18-month study period. In addition, an increase in the number of spots and deterioration in skin color tone uniformity during the 18-month non-sunscreen application period was expected. These results indicated that pigmentation spots respond better to treatment with a more noticeable change in appearance than wrinkles. It is thought that the effect of sunscreen on the prevention of pigmentation spots' formation is more developed and easily detectable than wrinkle formation in Japanese.

\section{Limitations}

This study was conducted with a small number of subjects with the lack of a control group in one center. This study design did not allow us to demonstrate high quality and clear evidence of the preventive effect of sunscreen on photoaging. Accordingly, a larger scale, designed study such as a randomized controlled trial needs to be performed for more solid proof. All the subjects in the study were over 60 years old and the average was $\sim 80$ years. The study was performed with only aged Japanese people. Therefore, the results are unlikely to be fully generalizable to the whole of the Japanese people.

\section{Conclusion}

In this study, we suggested an inhibitory effect on photoaging symptoms such as spots and skin color tone uniformity by application of the appropriate amount of sunscreen for a long period of time in Japanese people, similar to Caucasians.

To our knowledge, this is the first interventional clinical study suggesting the preventive effect of sunscreen on photoaging in Japanese people.

\section{Acknowledgments}

The abstract of this paper was presented at the 40th Annual Meeting of the Japanese Society for Investigative Dermatology Conference as a poster presentation with interim findings. The poster's abstract was published in the program of the 40th Annual Meeting of the Japanese Society for Investigative Dermatology.

\section{Disclosure}

Makoto Mizuno, Eiji Naru, and Koichi Kameyama are employees of KOSÉ Corporation, Japan. The authors report no other conflicts of interest in this work.

\section{References}

1. Boyd AS, Naylor M, Cameron GS, Pearse AD, Gaskell SA, Neldner KH The effects of chronic sunscreen use on the histologic changes of dermatoheliosis. J Am Acad Dermatol. 1995;33(6):941-946.

2. Green AC, Hughes MC, McBride P, Fourtanier A. Factors associated with premature skin aging (photoaging) before the age of 55: a populationbased study. Dermatology. 2011;222(1):74-80. 
3. Hughes MC, Williams GM, Baker P, Green AC. Sunscreen and prevention of skin aging: a randomized trial. Ann Intern Med. 2013;158(11): 781-790.

4. Food and Drug Administration H. Labeling and Effectiveness Testing; Sunscreen Drug Products for Over-the-Counter Human Use. US: Federal Register; 2011. Available from: https://www.gpo.gov/fdsys/pkg/ FR-2011-06-17/pdf/2011-14766.pdf. Accessed February 21, 2016.

5. The commission of the European communities [webpage on the Internet]. Commission Recommendation of 22 September 2006 on the efficacy of sunscreen products and the claims made relating thereto (notified under document number C(2006) 4089) (Text with EEA relevance). Official Journal of the European Union; 2006. Available from: http://eur-lex. europa.eu/legal-content/EN/TXT/?uri=CELEX\%3 A32006H0647. Accessed February 21, 2016.

6. Hillebrand GG, Miyamoto K, Schnell B, Ichihashi M, Shinkura R, Akiba S. Quantitative evaluation of skin condition in an epidemiological survey of females living in northern versus southern Japan. J Dermatol Sci. 2001;27 (Suppl 1):S42-S52.

7. Asakura K, Nishiwaki Y, Milojevic A, et al. Lifestyle factors and visible skin aging in a population of Japanese elders. J Epidemiol. 2009;19(5):251-259.

8. Tsukahara K, Fujimura T, Yoshida Y, et al. Comparison of age-related changes in wrinkling and sagging of the skin in Caucasian females and in Japanese females. J Cosmet Sci. 2004;55(4):351-371.

9. Perner D, Vierkotter A, Sugiri D, et al. Association between sunexposure, smoking behaviour and plasma antioxidant levels with the different manifestation of skin ageing signs between Japanese and German women--a pilot study. J Dermatol Sci. 2011;62(2):138-140.

10. Tsukahara K, Sugata K, Osanai O, et al. Comparison of age-related changes in facial wrinkles and sagging in the skin of Japanese, Chinese and Thai women. J Dermatol Sci. 2007;47(1):19-28.

11. Tsuchida T, Koga $H$, Uhara $H$, et al. Guidelines for diagnosis and treatment of skin malignant tumor in Japan. 2nd edition. Nihon Hifuka Gakkai Zasshi. 2015;125(1):5-75. Japanese.

12. Kunimoto K, Uede M, Mizuno M, Furukawa F, Yamamoto Y. The continued use of sunscreen prevents the development of actinic keratosis in aged Japanese subjects. Exp Dermatol. Suppl. In press 2016.

13. Fitzpatrick TB. The validity and practicality of sun-reactive skin types I through VI. Arch Dermatol. 1988;124(6):869-871.

14. Bremmer HJ. Prud'homme de Lodder LC, van Engelen JG. Cosmetics Fact Sheet To assess the risks for the consumer Updated version for ConsExpo 4. RIVM. 2006;320104001:1-77.

15. Sato J, Denda M, Nakanishi J, Koyama J. Dry condition affects desquamation of stratum corneum in vivo. J Dermatol Sci. 1998; 18(3):163-169.

16. Sato J, Yanai M, Hirao T, Denda M. Water content and thickness of the stratum corneum contribute to skin surface morphology. Arch Dermatol Res. 2000;292(8):412-417.

17. Pons-Guiraud A. Dry skin in dermatology: a complex physiopathology. J Eur Acad Dermatol Venereol. 2007;21 (Suppl 2):1-4.

18. Tezuka T. [Senile changes in epidermis; electron microscopic changes seen in the epidermis of xerosis senilis]. Nihon Hifuka Gakkai Zasshi. 1981;91(12):1299-1303. Japanese.

19. Thaipisuttikul Y. Pruritic skin diseases in the elderly. J Dermatol. 1998;25(3):153-157.

20. Hara M, Kikuchi K, Watanabe M, et al. Senile xerosis: Functional, morphological, and biochemical studies. J Geriatric Dermatol. 1993;1:111-120.

21. Potts RO, Buras EM Jr, Chrisman DA Jr. Changes with age in the moisture content of human skin. J Invest Dermatol. 1984;82(1):97-100.

22. Pillai S, Oresajo C, Hayward J. Ultraviolet radiation and skin aging: roles of reactive oxygen species, inflammation and protease activation, and strategies for prevention of inflammation-induced matrix degradation - a review. Int J Cosmet Sci. 2005;27(1):17-34.

23. Khansari N, Shakiba Y, Mahmoudi M. Chronic inflammation and oxidative stress as a major cause of age-related diseases and cancer. Recent Pat Inflamm Allergy Drug Discov. 2009;3(1):73-80.
24. Federico A, Morgillo F, Tuccillo C, Ciardiello F, Loguercio C. Chronic inflammation and oxidative stress in human carcinogenesis. Int $J$ Cancer. 2007;121(11):2381-2386.

25. Fluhr JW, Darlenski R, Surber C. Glycerol and the skin: holistic approach to its origin and functions. Br J Dermatol. 2008;159(1):23-34.

26. Batt MD, Davis WB, Fairhurst E, et al. Changes in the physical properties of the stratum corneum following treatment with glycerol. $J$ Soc Cosmet Chem. 1988;39:367-381.

27. Olsen EA, Abernethy ML, Kulp-Shorten C, et al. A double-blind, vehicle-controlled study evaluating masoprocol cream in the treatment of actinic keratoses on the head and neck. J Am Acad Dermatol. 1991;24(5 Pt 1):738-743.

28. Rivers JK, Arlette J, Shear N, Guenther L, Carey W, Poulin Y. Topical treatment of actinic keratoses with $3.0 \%$ diclofenac in $2.5 \%$ hyaluronan gel. Br J Dermatol. 2002;146(1):94-100.

29. de Berker D, McGregor JM, Hughes BR; British Association of Dermatologists Therapy Guidelines and Audit Subcommittee. Guidelines for the management of actinic keratoses. $\mathrm{Br} J$ Dermatol. 2007;156(2):222-230.

30. Lim SH, Kim SM, Lee YW, Ahn KJ, Choe YB. Change of biophysical properties of the skin caused by ultraviolet radiation-induced photodamage in Koreans. Skin Res Technol. 2008;14(1):93-102.

31. Kottner J, Lichterfeld A, Blume-Peytavi U. Transepidermal water loss in young and aged healthy humans: a systematic review and meta-analysis. Arch Dermatol Res. 2013;305(4):315-323.

32. Gilchrest BA. Skin aging and photoaging: an overview. $J$ Am Acad Dermatol. 1989;21(3 Pt 2):610-613.

33. Fisher GJ, Kang S, Varani J, et al. Mechanisms of photoaging and chronological skin aging. Arch Dermatol. 2002;138(11): $1462-1470$

34. Lock-Andersen J, Therkildsen P, de Fine Olivarius F, et al. Epidermal thickness, skin pigmentation and constitutive photosensitivity. Photodermatol Photoimmunol Photomed. 1997;13(4):153-158.

35. Lubowe II. Treatment of the aging skin by dermatologic methods. J Am Geriatr Soc. 1976;24(1):25-28.

36. Hashizume H. Skin aging and dry skin. J Dermatol. 2004;31(8): 603-609.

37. Nolan K, Marmur E. Moisturizers: reality and the skin benefits. Dermatol Ther. 2012;25(3):229-233.

38. Neale R, Williams G, Green A. Application patterns among participants randomized to daily sunscreen use in a skin cancer prevention trial. Arch Dermatol. 2002;138(10):1319-1325.

39. Faurschou A, Wulf HC. The relation between sun protection factor and amount of sunscreen applied in vivo. Br J Dermatol. 2007;156(4):716-719.

40. Reich A, Harupa M, Bury M, Chrzaszcz J, Starczewska A. Application of sunscreen preparations: a need to change the regulations. Photodermatol Photoimmunol Photomed. 2009;25(5):242-244.

41. Kim SM, Oh BH, Lee YW, Choe YB, Ahn KJ. The relation between the amount of sunscreen applied and the sun protection factor in Asian skin. J Am Acad Dermatol. 2010;62(2):218-222.

42. Teramura T MM, Asano H, Naito N, Arakane K, Miyachi Y. Relationship between sun-protection factor and application thickness in high-performance sunscreen: double application of sunscreen is recommended. Clin Exp Dermatol. 2012;37(8):904-908.

43. Peris K, Neri L, Fargnoli MC, Pellacani G. Physicians' concerns towards prescription adherence and treatment effectiveness in the clinical management of actinic keratosis. G Ital Dermatol Venereol. 2014;149(2):193-198.

44. Esmann S, Jemec GB. Patients' perceptions of topical treatments of actinic keratosis. J Dermatolog Treat. 2014;25(5):375-379.

45. Williams LK, Pladevall M, Xi H, et al. Relationship between adherence to inhaled corticosteroids and poor outcomes among adults with asthma. J Allergy Clin Immunol. 2004;114(6):1288-1293.

46. Devaux S, Castela A, Archier E, et al. Adherence to topical treatment in psoriasis: a systematic literature review. J Eur Acad Dermatol Venereol. 2012;26 (Suppl 3):61-67. 
47. Thompson SC, Jolley D, Marks R. Reduction of solar keratoses by regular sunscreen use. N Engl J Med. 1993;329(16):1147-1151.

48. Green A, Williams G, Neale R, et al. Daily sunscreen application and betacarotene supplementation in prevention of basal-cell and squamouscell carcinomas of the skin: a randomised controlled trial. Lancet. 1999;354(9180):723-729.
49. Traianou A, Ulrich M, Apalla Z, et al. Risk factors for actinic keratosis in eight European centres: a case-control study. Br J Dermatol. 2012; 167 (Suppl 2):36-42.

\section{Publish your work in this journal}

Clinical, Cosmetic and Investigational Dermatology is an international, peer-reviewed, open access, online journal that focuses on the latest clinical and experimental research in all aspects of skin disease and cosmetic interventions. All areas of dermatology will be covered; contributions will be welcomed from all clinicians and basic science researchers globally. This journal is indexed on CAS. The manuscript management system is completely online and includes a very quick and fair peer-review system, which is all easy to use. Visit http://www.dovepress.com/testimonials.php to read real quotes from published authors.

Submit your manuscript here: http://www.dovepress.com/clinical-cosmetic-and-investigational-dermatology-journal 